\title{
Observer-Based Anomaly Detection of Synchronous Generators for Power Systems Monitoring
}

\author{
Georgios Anagnostou ${ }^{(0)}$, Member, IEEE, Francesca Boem ${ }^{(0)}$, Stefanie Kuenzel ${ }^{\circledR}$, Member, IEEE, \\ Bikash C. Pal ${ }^{\circledR}$, Fellow, IEEE, and Thomas Parisini ${ }^{\circledR}$, Fellow, IEEE
}

\begin{abstract}
This paper proposes a rigorous anomaly detection scheme, developed to spot power system operational changes which are inconsistent with the models used by operators. This novel technique relies on a state observer, with guaranteed estimation error convergence, suitable to be implemented in real time, and it has been developed to fully address this important issue in power systems. The proposed method is fitted to the highly nonlinear characteristics of the network, with the states of the nonlinear generator model being estimated by means of a linear time-varying estimation scheme. Given the reliance of the existing dynamic security assessment tools in industry on nominal power system models, the suggested methodology addresses cases when there is deviation from assumed system dynamics, enhancing operators' awareness of system operation. It is based on a decision scheme relying on analytical computation of thresholds, not involving empirical criteria which are likely to introduce inaccurate outcomes. Since falsealarms are guaranteed to be absent, the proposed technique turns out to be very useful for system monitoring and control. The effectiveness of the anomaly detection algorithm is shown through detailed realistic case studies in two power system models.
\end{abstract}

Index Terms-Fault detection, observers, power system dynamics, power system monitoring, synchronous generators.

\section{NOMENCLATURE}

$\alpha \quad$ Difference between rotor angle and HV bus voltage phase in rad.

$\bar{\epsilon} \quad$ Vector of maximum state estimation errors.

Manuscript received May 8, 2017; revised September 12, 2017; accepted October 27, 2017. Date of publication January 1, 2018; date of current version June 18, 2018. This work was supported in part by the U.K.-China initiative in Stability and Control of Power Networks With Energy Storage (STABLE-NET) funded by Research Councils U.K. Energy Programme under Grant EP/L014343/1), and in part by the European Union's Horizon 2020 research and innovation programme (KIOS CoE) under Grant 739551. Paper no. TPWRS-00683-2017. (Corresponding author: Bikash C. Pal.)

G. Anagnostou and B. C. Pal are with the Department of Electrical and Electronic Engineering, Imperial College London, London SW7 2AZ, U.K. (e-mail: georgios.anagnostou11@imperial.ac.uk; b.pal@imperial.ac.uk).

F. Boem is with the Department of Electrical and Electronic Engineering, Imperial College London, London SW7 2AZ, U.K., and also with the KIOS Research and Innovation Center of Excellence, University of Cyprus, Nicosia 1678, Cyprus (e-mail: f.boem@imperial.ac.uk).

S. Kuenzel is with the Department of Electronic Engineering, Royal Holloway, University of London, Egham TW20 0EX, U.K. (e-mail: stefanie. kuenzel@ rhul.ac.uk).

T. Parisini is with the Department of Electrical and Electronic Engineering, Imperial College London, London SW7 2AZ, U.K., with the Department of Engineering and Architecture, University of Trieste, TS 34127, Italy, and also with the KIOS Research and Innovation Center of Excellence, University of Cyprus, Nicosia 1678, Cyprus (e-mail: t.parisini@imperial.ac.uk).

Color versions of one or more of the figures in this paper are available online at http://ieeexplore.ieee.org.

Digital Object Identifier 10.1109/TPWRS.2017.2771278
Vector of maximum measured input noise values. Vector of maximum measurement noise values. Transformer off-nominal ratio. Vector of maximum process disturbance values. Rotor angle in rad.

Vector of state estimation errors.

Column vector of measured input noise variables.

$\omega, \omega_{B} \quad$ Rotor speed in p.u. and its base value in $\mathrm{rad} / \mathrm{s}$.

$\phi_{I y} \quad$ Measured stator current phase.

$\Phi \quad$ Discrete-time form of system transition matrix.

$\psi_{1 d} \quad$ Subtransient emf due to $d$-axis damper coil in p.u.

$\psi_{2 q} \quad$ Subtransient emf due to $q$-axis damper coil in p.u.

$\mathbf{I}_{n} \quad$ Identity $\mathrm{n} \times \mathrm{n}$ matrix.

$\theta \quad$ HV bus voltage phase in rad.

$\tilde{u} \quad$ Column vector of noisy inputs.

$v_{f} \quad$ Measurement noise associated with $f_{\text {sys }}$.

$v_{I} \quad$ Measurement noise associated with $I_{y}$.

$v_{\phi_{I}} \quad$ Measurement noise associated with $\phi_{I y}$.

$v, \tilde{v} \quad$ Vectors of measurement noise variables.

$A, B \quad$ Discrete-time state and input matrices.

$C, D \quad$ Discrete-time output and feedforward input matrices.

$D_{r} \quad$ Rotor damping constant in p.u.

$E_{d}^{\prime} \quad$ Transient emf due to flux in q-axis damper coil in p.u.

$E_{q}^{\prime} \quad$ Transient emf due to field flux linkages in p.u.

$E_{f d} \quad$ Generator field excitation voltage in p.u.

$f \quad$ Discrete-time form of system state equations.

$f_{\theta} \quad$ Rate of change of the HV bus voltage phase in p.u.

$f_{v} \quad$ Noise term of the measured value of $f_{\theta}$ in p.u.

$f_{y} \quad$ Measured value of $f_{\theta}$ in p.u.

$f_{\text {sys }} \quad$ Measured system frequency and its associated noise.

$G \quad$ Observability Gramian matrix.

$h \quad$ Discrete-time form of measurement output equations.

I Stator current magnitude in p.u.

$I_{y} \quad$ Measured stator current magnitude.

$K \quad$ Observer filtering matrix.

$k \quad$ Simulation time step.

$K_{A} \quad$ Automatic voltage regulator gain.

$K_{d 1} \quad$ Ratio $\left(X_{d}^{\prime \prime}-X_{l s}\right) /\left(X_{d}^{\prime}-X_{l s}\right)$.

$K_{d 2} \quad$ Ratio $\left(X_{d}^{\prime}-X_{d}^{\prime \prime}\right) /\left(X_{d}^{\prime}-X_{l s}\right)$.

$K_{q 1} \quad$ Ratio $\left(X_{q}^{\prime \prime}-X_{l s}\right) /\left(X_{q}^{\prime}-X_{l s}\right)$

$K_{q 2} \quad$ Ratio $\left(X_{q}^{\prime}-X_{q}^{\prime \prime}\right) /\left(X_{q}^{\prime}-X_{l s}\right)$.

$M \quad$ Inertia constant in p.u.

$P_{y}, v_{P} \quad$ Measured active power value and its associated noise.

$Q_{y}, v_{Q}$ Measured reactive power value and its associated noise. 


$\begin{array}{ll}r, \bar{r} & \text { Vectors of residuals and thresholds. } \\ R_{s} & \text { Armature resistance in p.u. } \\ R_{T} & \text { Transformer winding resistance in p.u. } \\ T_{d 0}^{\prime} & d \text {-axis transient time constant. } \\ T_{q 0}^{\prime} & q \text {-axis transient time constant. } \\ T_{0} & \text { Simulation time step. } \\ T_{A} & \text { Automatic voltage regulator time constant. } \\ T_{m}, T_{e} & \text { Mechanical and electrical torque inputs in p.u. } \\ T_{d 0}^{\prime \prime} & d \text {-axis subtransient time constant in s. } \\ T_{q 0}^{\prime \prime} & q \text {-axis subtransient time constant in s. } \\ V^{\prime} & \text { HV bus voltage magnitude in p.u. } \\ V_{v} & \text { Noise term of the measured value of } V \text { in p.u. } \\ V_{t} & \text { Stator voltage magnitude in p.u. } \\ V_{y} & \text { Measured value of } V \text { in p.u. } \\ V_{r e f} & \text { Automatic voltage regulator reference constant in p.u. } \\ w, \tilde{w} & \text { Vectors of process disturbances. } \\ x, \hat{x} & \text { Vectors of system states and state estimates. } \\ X_{d}^{\prime}, X_{q}^{\prime} & d \text {-axis and } q \text {-axis transient reactances in p.u. } \\ X_{d}, X_{q} & d \text {-axis and } q \text {-axis synchronous reactances in p.u. } \\ X_{T} & \text { Transformer leakage reactance in p.u. } \\ X_{d}^{\prime \prime}, X_{q}^{\prime \prime} & d \text {-axis and } q \text {-axis subtransient reactances in p.u. } \\ X_{l s} & \text { Armature leakage reactance in p.u. } \\ y, \hat{y} & \text { Vectors of measurements and output predictions. } \\ Z_{T} & \text { Transformer series impedance in p.u. }\end{array}$

\section{INTRODUCTION}

M ODERN power networks are undergoing considerable operational and structural modifications, resulting from significant technology advances with respect to forms of electric power generation and communications infrastructure, let alone the energy market liberalisation [1], [2]. Power system complexity increases, given the current trends. Specifically, advanced control systems associated with newly integrated system components may give rise to unpredictable system behaviour of nonlinear nature. Moreover, aging equipment, given the longstanding power network operation, is prone to failures, whereas, lack of investments in transmission systems may lead to stressed system operation [2]. In this context, it is widely recognised that wide area monitoring systems (WAMS) contribute to operators' knowledge of system's operational status, and dynamic state estimation (DSE) may prove to be a very useful tool [3].

Real-time DSE is model based, thus, good knowledge of the power system dynamic model is essential to obtain accurate results. In this context, given power networks' high complexity, methods detecting deviation from routine conditions or 'nominal' models play a significant role in enhancing operators' awareness of the system operation. Such anomaly detection techniques have been reported in power systems literature, engaging Kalman filter variants, and relying on thresholds, the values of which are typically based on empirical criteria [4][9]. In this respect, Kalman filter utilization requires knowledge and assumptions regarding noise probability distributions (e.g. Gaussianity), while divergence issues are likely to arise when there is a high level of mismatch between the assumed estimation system model and the real one [10].

On the other hand, observer-based anomaly detection techniques constitute a significant class of methods identifying deviations from nominal models, being popular in various research areas [11]-[18]. Observers are developed based on the principle of guaranteed estimation error convergence (whereas Kalman filter based estimators primarily rely on the trace minimization of the state error covariance) [19], [20]. There have been several observer based research studies, involving power system models requiring many assumptions and simplifications [21], [22]; in cases where more advanced power system models are utilized, they are linearised with respect to one specific operating point, with the observer's filtering matrix term being optimized with reference to this particular point [23]-[26], restricting the applicability of the observer in a continuously changing environment like operation of a power network.

To address these issues, the work conducted in [27] is extended to consider nonlinear output equations, leading to the development of a discrete-time observer, based on time-varying linearisation with respect to the estimate at every time step, in a similar approach as in Extended Kalman filtering (EKF) [10]. The filtering term, guaranteeing the observer's convergence, changes at every time step, and its computation is simple and suitable for real-time implementations. This research effort leads to the following main contributions:

- to propose a novel observer in the context of realistic power system models;

- to establish an observer-based anomaly detection technique for models with nonlinear output equations;

- to formulate a systematic approach for threshold calculation, guaranteeing the absence of false alarms, unlike the case where empirical criteria are used.

The paper is organised as follows: In the next section, the synchronous generator models dealt with in this research are presented and thoroughly analysed. Section III includes the analysis of the observer-based anomaly detection algorithm. In Section IV, the proposed methodology is applied to two study systems; the 9-bus, 3-machine system used in [28] and the IEEE benchmark 68-bus, 16-machine system, corresponding to the New England (NETS) and New York (NYPS) power systems, along with three neighbouring areas [29]. Section $\mathrm{V}$ gives some concluding remarks.

\section{Synchronous GENERATOR Estimation Models}

\section{A. Model Development - State Equations}

Synchronous generator modelling is one of the basic requirements of power system dynamic modelling. Depending on the targets of each research and the amount of information available about the modelling detail, various models have been reported in literature [28], [30]. In the context of multi-machine systems, decentralisation has gained popularity, since it reduces the computational burden and enables the estimation procedure to be conducted on a local basis [31]-[35]. The decentralisation procedure relies on system partitioning (in terms of the estimation calculations) and the use of measured inputs, which are measurements on the 'boundary' of the assumed subsystem [31], [32]. This partitioning based approach is usually called 'playback process', which is also useful in generator model validation procedures [36]. Here, the 'boundary' is the high voltage (HV) side of the transformer connected to the terminal bus of 


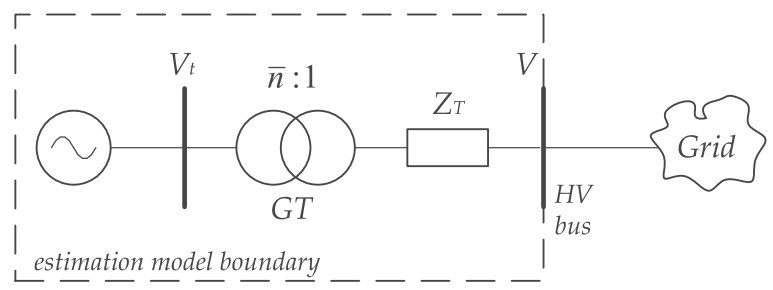

Fig. 1. Estimation model boundary in the context of a multimachine system.

the respective generator (henceforth termed as 'HV bus'), to enable local anomaly detection based on measurements at the bus which corresponds to this side of the transformer, as in [36]. The assumed estimation model boundary is depicted in Fig. 1. In several power system studies, the noise component of the measured inputs has been taken into account in the context of the analysis [32], [33], whereas, in some other studies, these inputs are considered as noise-free [34], [35], [37]. Furthermore, the consideration of different measured inputs leads to different decentralised synchronous generator models, with diverse models having been utilized for this purpose [32]-[35], [37]. Two synchronous generator models have been used in the context of the case studies here, the transient and the subtransient models [28], [38], [39]. Both models are valid for transient conditions, with the subtransient being more detailed and capturing phenomena taking place immediately after an event occurrence [30]. The subtransient synchronous generator model used here, including the respective transformer model elements, is described by the following equations [28], [38]:

$$
\begin{aligned}
\dot{\alpha}= & \omega_{B}\left(\omega-1-f_{\theta}\right), \\
\dot{\omega}= & \frac{1}{M}\left[T_{m}-T_{e}-D_{r}(\omega-1)\right], \\
\dot{E}_{q}^{\prime}= & \frac{1}{T_{d 0}^{\prime}}\left\{E_{f d}-E_{q}^{\prime}-\left(X_{d}-X_{d}^{\prime}\right)\left[-I_{d}\right.\right. \\
& \left.\left.-\frac{K_{d 2}}{X_{d}^{\prime}-X_{l s}}\left(\psi_{1 d}-\left(X_{d}^{\prime}-X_{l s}\right) I_{d}-E_{q}^{\prime}\right)\right]\right\}, \\
\dot{E}_{d}^{\prime}= & \frac{1}{T_{q 0}^{\prime}}\left\{-E_{d}^{\prime}-\left(X_{q}-X_{q}^{\prime}\right)\left[I_{q}\right.\right. \\
& \left.\left.-\frac{K_{q 2}}{X_{q}^{\prime}-X_{l s}}\left(-\psi_{2 q}+\left(X_{q}^{\prime}-X_{l s}\right) I_{q}-E_{d}^{\prime}\right)\right]\right\}, \\
\dot{\psi}_{2 q}= & \frac{1}{T_{q 0}^{\prime \prime}}\left[-\psi_{2 q}-E_{d}^{\prime}+\left(X_{q}^{\prime}-X_{l s}\right) I_{q}\right], \\
\dot{\psi}_{1 d}= & \frac{1}{T_{d 0}^{\prime \prime}}\left[-\psi_{1 d}+E_{q}^{\prime}+\left(X_{d}^{\prime}-X_{l s}\right) I_{d}\right], \\
\dot{E}_{f d}= & \frac{1}{T_{A}}\left[K_{A}\left(V_{r e f}-V_{t}\right)-E_{f d}\right],
\end{aligned}
$$

and

$$
\begin{aligned}
T_{e}= & K_{q 1} E_{d}^{\prime} I_{d}+K_{d 1} E_{q}^{\prime} I_{q} \\
& +\left(X_{d}^{\prime \prime}-X_{q}^{\prime \prime}\right) I_{d} I_{q}+K_{d 2} \psi_{1 d} I_{q}-K_{q 2} \psi_{2 q} I_{d} \\
{\left[\begin{array}{c}
I_{d} \\
I_{q}
\end{array}\right]=} & Z_{g t r}^{-1}\left[\begin{array}{cc}
R_{s} & -X_{q}^{\prime \prime} \\
X_{d}^{\prime \prime} & R_{s}
\end{array}\right]\left[\begin{array}{l}
K_{q 1} E_{d}^{\prime}-K_{q 2} \psi_{2 q}-\bar{n} V_{d} \\
K_{d 1} E_{q}^{\prime}+K_{d 2} \psi_{1 d}-\bar{n} V_{q}
\end{array}\right],
\end{aligned}
$$

$$
\begin{aligned}
Z_{g t r} & =\mathbf{I}_{2}+\bar{n}^{2}\left[\begin{array}{cc}
R_{s} & X_{q}^{\prime \prime} \\
-X_{d}^{\prime \prime} & R_{s}
\end{array}\right]^{-1}\left[\begin{array}{cc}
R_{T} & X_{T} \\
-X_{T} & R_{T}
\end{array}\right], \\
{\left[\begin{array}{c}
V_{t d} \\
V_{t q}
\end{array}\right] } & =\bar{n}^{2}\left[\begin{array}{cc}
R_{T} & X_{T} \\
-X_{T} & R_{T}
\end{array}\right]\left[\begin{array}{c}
I_{d} \\
I_{q}
\end{array}\right]+\bar{n}\left[\begin{array}{c}
V_{d} \\
V_{q}
\end{array}\right], \\
V_{t} & =\sqrt{V_{t q}^{2}+V_{t d}^{2}} \\
V_{q} & =V \cos \alpha \\
V_{d} & =-V \sin \alpha \\
V & =V_{y}-V_{v} \\
f_{\theta} & =f_{y}-f_{v} .
\end{aligned}
$$

To obtain the discrete form of these equations, it can be assumed that $\dot{x}=\left(x_{k}-x_{k-1}\right) / T_{0}$, where $T_{0}$ is the simulation time step. This model is built based on the principle that d-axis leads q-axis. Similarly to the analysis in [33], the internal rotor angle $(\alpha)$ is used instead of the rotor angle $(\delta)$, since the utilization of the latter relies on the multi-machine context, requiring the knowledge of the global reference frame angle, which is impossible when estimation is performed based on local information and measurements only. Here, the phase angles of all quantities are defined with respect to the HV bus voltage phasor. With regard to this, the internal rotor angle $(\alpha)$ replaces the rotor angle $(\delta)$ in this decentralised context, as carried out in [33], defined as $\alpha=\delta-\theta$, with (1) characterizing its dynamics [33]. The rate of change of the HV bus voltage phase is approximated by the equation below (divided by $\omega_{B}$, to obtain the p.u. value) [28]:

$$
f_{\theta k} \approx \frac{\theta_{k}-\theta_{k-1}}{\omega_{B} T_{0}}
$$

Analogously to the procedure described in [33], the measured inputs used are the $\mathrm{HV}$ bus voltage magnitude $\left(V_{y}\right)$ and the rate of change of its phase $\left(f_{y}\right)$. Thus, in the context of the synchronous generator subtransient model, the state vector has the following form:

$$
x=\left[\alpha \omega E_{q}^{\prime} E_{d}^{\prime} \psi_{2 q} \psi_{1 d} E_{f d}\right]^{\top} .
$$

Also, the noisy input vector is:

$$
\tilde{u}=\left[\begin{array}{llll}
T_{m} & V_{\text {ref }} & V_{y} & f_{y}
\end{array}\right]^{\top},
$$

whereas:

$$
\begin{aligned}
& u=\left[\begin{array}{llll}
T_{m} & V_{\text {ref }} & V & f_{\theta}
\end{array}\right]^{\top}, \\
& \nu=\left[\begin{array}{llll}
0 & 0 & V_{v} & f_{v}
\end{array}\right]^{\top},
\end{aligned}
$$

where $u, \nu$ correspond to the noise-free and noise components of the noisy input vector, respectively (i.e. $\tilde{u}=u+\nu$ ). It can be noted that the first two elements of $\nu$ are equal to 0 , since $T_{m}$ and $V_{\text {ref }}$ are considered to be perfectly known.

Further to the subtransient model, the transient model can be obtained by considering $X_{q}^{\prime \prime}=X_{q}^{\prime}$ and $X_{d}^{\prime \prime}=X_{d}^{\prime}$, meaning that $K_{q 1}=K_{d 1}=1$ and $K_{q 2}=K_{d 2}=0$; therefore, in this case, the state vector does not include $\psi_{2 q}$ and $\psi_{1 d}((5),(6)$ are omitted). 


\section{B. Output Equations}

This work considers a decentralized generator model, with a Phasor Measurement Unit (PMU) at the HV side of the transformer which comes after the generator terminal bus. The current magnitude $\left(I_{y}\right)$, its phase with reference to the $\mathrm{HV}$ bus voltage phasor $\left(\phi_{I y}\right)$, the active power output $\left(P_{y}\right)$ and the reactive power output $\left(Q_{y}\right)$ at the $\mathrm{HV}$ bus are the measurement outputs. These are governed by the following equations:

$$
\begin{aligned}
I_{y}= & \bar{n} \sqrt{I_{q}^{2}+I_{d}^{2}}+v_{I} \\
\phi_{I y}= & \alpha+\arctan \left(\frac{I_{d}}{I_{q}}\right)+v_{\phi_{I}} \\
P_{y}= & K_{q 1} E_{d}^{\prime} I_{d}+K_{d 1} E_{q}^{\prime} I_{q}+\left(X_{d}^{\prime \prime}-X_{q}^{\prime \prime}\right) I_{d} I_{q}+K_{d 2} \psi_{1 d} I_{q} \\
& -K_{q 2} \psi_{2 q} I_{d}-\left(I_{d}^{2}+I_{q}^{2}\right)\left(R_{s}+\bar{n}^{2} R_{T}\right)+v_{P} \\
Q_{y}= & K_{q 1} E_{d}^{\prime} I_{q}-K_{q 2} \psi_{2 q} I_{q}-\left(X_{q}^{\prime \prime}+\bar{n}^{2} X_{T}\right) I_{q}^{2} \\
& -\left(X_{d}^{\prime \prime}+\bar{n}^{2} X_{T}\right) I_{d}^{2}-K_{d 1} E_{q}^{\prime} I_{d}-K_{d 2} \psi_{1 d} I_{d}+v_{Q}
\end{aligned}
$$

where $I_{q}, I_{d}$ are given by (9), whereas $v_{I}, v_{\phi_{I}}$ are the measurement noise terms, associated with $I_{y}$ and $\phi_{I y}$, respectively, $P_{y}$ and $Q_{y}$ are the active and reactive power, respectively, measured at the HV substation of the transformer of the corresponding generator, and $v_{P}, v_{Q}$ are their associated noise terms, respectively.

Frequency measurement $\left(f_{s y s_{y}}\right)$ has also been considered, since it is closely related to speed [40], and its p.u. value is:

$$
f_{s y s_{y}}=\omega+v_{f},
$$

where $v_{f}$ is the associated measurement noise. Therefore, the output measurement vector is:

$$
y=\left[\begin{array}{lllll}
f_{\text {sys }} & I_{y} & \phi_{I y} & P_{y} & Q_{y}
\end{array}\right]^{\top} .
$$

\section{Anomaly Detection Methodology}

\section{A. The Anomaly Detection Logic}

The proposed anomaly detection method is inspired by the work conducted in [27], which has been significantly extended to consider the nonlinear output equations. (1)-(16) and (22)(26) constitute the nonlinear synchronous generator state space model, which can be discretized and described by the following general discrete-time model:

$$
\begin{aligned}
x_{k+1} & =f\left(x_{k}, u_{k}\right)+\tilde{w}_{k}, \\
y_{k} & =h\left(x_{k}, u_{k}\right)+\tilde{v}_{k},
\end{aligned}
$$

where $f$ represents the discretized system state (1)-(16) at time step $k, h$ corresponds to the discretized output measurement (22)-(26), $\tilde{w}_{k}$ is the process disturbance vector accounting for modelling uncertainty, whereas $\tilde{v}_{k}$ is the output measurement noise vector.

In order for anomalies in the system to be detected, a model based decision scheme is proposed. A prediction $\hat{y}_{k}$ of the output measurements $y_{k}$ is computed with reference to the nominal model (28). The prediction error $r_{k}=y_{k}-\hat{y}_{k}$ is compared with a suitably defined threshold to decide whether the current prediction error is just caused by the process and the output disturbances or also by the additional influence of an anomaly causing a significant discrepancy between the measured output and the one predicted via the nominal model.

The following time-varying observer is defined to compute the output prediction:

$$
\begin{aligned}
\hat{x}_{k+1} & =A_{k} \hat{x}_{k}+B_{k} \tilde{u}_{k}+\tilde{b}_{k}+K_{k}\left(y_{k}-\hat{y}_{k}\right), \\
\hat{y}_{k} & =C_{k} \hat{x}_{k}+D_{k} \tilde{u}_{k}+\tilde{c}_{k}, \\
\tilde{u}_{k} & =u_{k}+\nu_{k},
\end{aligned}
$$

with $A_{k}, B_{k}, C_{k}, D_{k}, \tilde{b}_{k}, \tilde{c}_{k}$ defined as:

$$
\begin{aligned}
A_{k} & =\left.\frac{\partial f}{\partial x_{k}}\right|_{\hat{x}_{k}}, B_{k}=\left.\frac{\partial f}{\partial u_{k}}\right|_{\hat{x}_{k}}, C_{k}=\left.\frac{\partial h}{\partial x_{k}}\right|_{\hat{x}_{k}}, D_{k}=\left.\frac{\partial h}{\partial u_{k}}\right|_{\hat{x}_{k}} \\
\tilde{b}_{k} & =f\left(\hat{x}_{k}, \tilde{u}_{k}\right)-A_{k} \hat{x}_{k}-B_{k} \tilde{u}_{k}, \\
\tilde{c}_{k} & =h\left(\hat{x}_{k}, \tilde{u}_{k}\right)-C_{k} \hat{x}_{k}-D_{k} \tilde{u}_{k},
\end{aligned}
$$

where $\hat{x}$ is the state estimation, and $K$ a matrix collecting the time-varying observer parameters. Matrix $K_{k}$ is computed at each time step as in [41], guaranteeing the convergence of the state estimation error $\epsilon_{k}=x_{k}-\hat{x}_{k}$ (see the theoretical analysis illustrated in the Appendix B).

The prediction error is compared component-by-component with a suitably defined threshold (see Section III-C). If, for at least one component, the residual crosses the corresponding threshold, this designates an anomaly (i.e., the measurements cannot be "explained" by the nominal dynamics). However, before the threshold analysis is presented, the system model (28) has to be formulated in an appropriate way with respect to the aforementioned observer equations, as illustrated in the following section.

\section{B. Reformulation of the Nominal Model}

From (1)-(16) and (22)-(26), it is clear that the synchronous generator's state and output equations are nonlinear. In order to allow dynamical analysis for the rigorous definition of a suitable anomaly detection threshold, the model (28) is reformulated as a linear time-varying model. For this purpose, the discrete-time state and output equations are linearised around the estimate at every time step (a similar approach is described in [10]), to obtain the prediction for the next time step. Thus, the previously presented synchronous generator models are rewritten according to the following discrete-time state space form:

$$
\begin{aligned}
x_{k+1} & =A_{k} x_{k}+B_{k} u_{k}+\tilde{b}_{k}+w_{k}, \\
y_{k} & =C_{k} x_{k}+D_{k} u_{k}+\tilde{c}_{k}+v_{k},
\end{aligned}
$$

where $w_{k}$ and $v_{k}$ also include the linearisation errors. In the context of the analysis conducted here, the process disturbance vector $w$ lies within a range of values corresponding to nominal operation, and, as far as the measured input and output measurement noises (i.e. $\nu$ and $v$, respectively) are concerned, they are characterized by maximum errors, as specified by standards, with which the measurement devices used have to comply, and 
in this case the IEEE Standard C37.118.1-2011 for phasor measurement units (PMUs) [42]. This means that:

$$
\left|w_{k}\right| \leq \bar{w}, \quad\left|\nu_{k}\right| \leq \bar{\nu}, \quad\left|v_{k}\right| \leq \bar{v} .
$$

If any of the above inequalities is violated, this denotes deviation from nominal system operation and, thus, it is considered as an anomaly.

\section{The Anomaly Detection Threshold}

The anomaly detection threshold is designed in order to act as an upper bound for the output estimation error in the presence of disturbances. The threshold is analytically designed so to guarantee the following: i) to always be an upper bound for the residual; ii) absence of false-alarms; iii) stability of the threshold. To derive a suitable threshold, the output estimation error is analysed:

$$
r_{k}=y_{k}-\hat{y}_{k}=C_{k} \epsilon_{k}-D_{k} \nu_{k}+v_{k} .
$$

Thanks to the result in Proposition B.1 (see Appendix B) guaranteeing the convergence of the estimator, and, from (31), the output estimation error (32) can be bounded at each time step component-wise using the triangular inequality:

$$
\left|r_{k}\right| \leq\left|C_{k}\right|\left|\epsilon_{k}\right|+\left|D_{k}\right| \bar{\nu}+\bar{v} .
$$

The state estimation error $\epsilon_{k}=x_{k}-\hat{x}_{k}$ is then analysed (see (38) in Appendix B). It can be written as:

$$
\epsilon_{k}=\Phi_{k, 0} \epsilon_{0}+\sum_{l=0}^{k-1} \Phi_{k, l+1} \tilde{d}_{l},
$$

where $\Phi_{k, 0}$ is the transition matrix from time 0 to time $k$ :

$$
\Phi_{k, 0}=\tilde{A}_{k-1} \tilde{A}_{k-2} \ldots \tilde{A}_{0},
$$

with $\tilde{A}_{k}=A_{k}-K_{k} C_{k}$ and $\tilde{d}_{l}=-B_{l} \nu_{l}+w_{l}-K_{l}\left(-D_{l} \nu_{l}+\right.$ $\left.v_{l}\right)$. Since the unforced system describing the dynamics of the estimation error (Appendix B - (35)) is exponentially asymptotically stable thanks to the result in Appendix B, in [43] it is proved that there exist $a>0$ and $\lambda \in[0,1]$ so that:

$$
\left\|\Phi_{k, 0}\right\| \leq a \lambda^{k} .
$$

Therefore, the state estimation error can be bounded by:

$$
\bar{\epsilon}_{k}=a \lambda^{k} \bar{\epsilon}_{0}+\sum_{l=0}^{k-2} a \lambda^{k-l-1} \bar{d}_{l}+\bar{d}_{k-1},
$$

where $\bar{\epsilon}_{0}$ is the initial estimation error bound, properly defined thanks to the knowledge of the system, and $\bar{d}_{l}=\left|B_{l}\right| \bar{\nu}+\bar{w}+$ $\left|K_{l}\right|\left(\left|D_{l}\right| \bar{\nu}+\bar{v}\right)$. As a consequence, we can define the anomaly detection threshold for the residual signal $r$ :

$$
\bar{r}_{k}:=\left|C_{k}\right| \bar{\epsilon}_{k}+\left|D_{k}\right| \bar{\nu}+\bar{v} .
$$

Thanks to the way it is designed, the following inequality is a necessary condition for the 'healthy' status of the monitored system:

$$
\left|r_{k}^{(i)}\right| \leq \bar{r}_{k}^{(i)}, \quad \forall i=1, \ldots, 5 .
$$

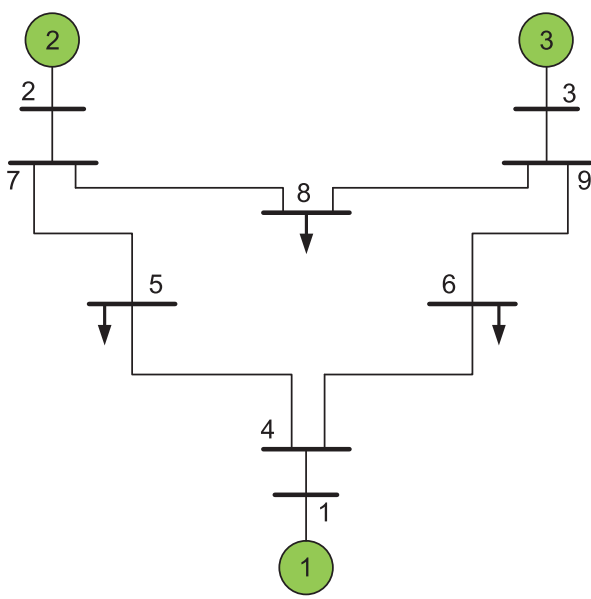

Fig. 2. 9-bus, 3-machine system.

It is sufficient that at least one component of the residual signal $\left|r^{(i)}\right|$ crosses the corresponding threshold $\bar{r}^{(i)}$ for at least one time-step to state that the monitored system cannot be explained by the nominal dynamics (30), i.e., something has changed in the dynamics of the system from its modelled dynamics.

\section{CASe Studies}

\section{A. 9-Bus, 3-Machine System}

The anomaly detection methodology has been applied to a 9-bus, 3-machine power system model, the details of which can be found in [28], and it is shown in Fig. 2. In this model, all synchronous machines are designed using their transient models, as previously presented, whereas, fast static exciters have been used for all the machines, described by (7), as opposed to the DC excitation systems which are utilized in [28], [39], to enable fast system response to contingencies [4]. With reference to (7), the parameter details of the excitation system used are $K_{A}=200$, and $T_{A}=0.01 \mathrm{~s}$, for all generators. Regarding the estimation procedure, IEEE Standards-compliant PMUs with reporting rate of 120 frames per second are used, to obtain measurements at the HV side of the transformer which comes after the terminal bus of the synchronous generator of interest. The maximum error of the measured voltage magnitude is $9 \cdot 10^{-3}$ p.u., and, the one corresponding to the voltage phase is $2 \cdot 10^{-3} \mathrm{rad}$, whereas the maximum errors for the measured active and reactive power values is $6 \cdot 10^{-3}$ p.u. This errors are set in accordance with the PMU related requirement of maximum $1 \%$ total vector error (TVE), dictated by the IEEE Standard C37.118.1-2011, blending together three possible sources of error for each phasor: phasor magnitude, angle and time synchronisation [42]. In the same context, the maximum frequency error is $0.005 \mathrm{~Hz}$ [42]. It has to be noted that the process disturbance is comprised by the noise coming from the measured inputs, and the estimation model is considered to be a highly accurate representation of the real one. Power system modelling is MATLAB/Simulink based, and all simulations last for $10 \mathrm{~s}$. Three case studies have been considered: 

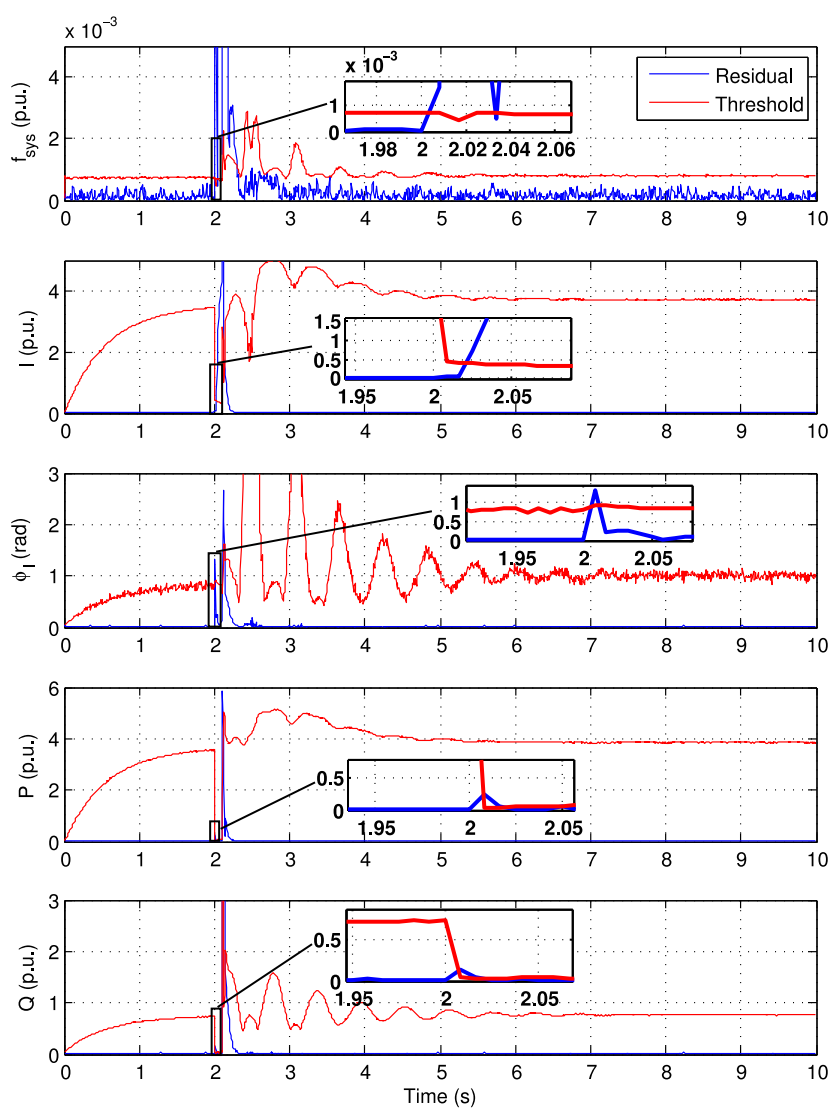

Fig. 3. Case Study 1A: Output residuals and thresholds for Gen. 3.

- Case Study 1A: A three-phase to ground fault occurs at bus 9 , at the time instant $t=2 \mathrm{~s}$, it is cleared after $100 \mathrm{~ms}$ and the line connecting buses 8 and 9 is tripped at the same time. The measurements are obtained at bus 9 , and the generator of interest is Gen. 3 .

- Case Study 1B: A step increase by 1 p.u. in $T_{m}$ of Gen. 2 occurs at the time instant $t=2 \mathrm{~s}$ and lasts for $1 \mathrm{~s}$, returning to its previous value afterwards. The measurements are obtained at bus 7 .

- Case Study 1C: The overexcitation limiter (OEL) of Gen. 1 gets activated at the time instant $t=3.34 \mathrm{~s}$. The OEL used is of takeover type, designed according to the error signal substitution scheme, the design characteristics and the block diagram of which can be found in [4]. This OEL type is likely to lower system stability margins, therefore, it is important for its activation to be detected by the proposed anomaly identification scheme [4]. The measurements are obtained at bus 4 .

The results showing the residual and the threshold values for all measurement outputs of the case studies $1 \mathrm{~A}, 1 \mathrm{~B}, 1 \mathrm{C}$, and Gens. 3, 2, 1, respectively, are illustrated in Figs. 3, 4 and 5, respectively. It can be clearly noticed that the proposed method is able to capture all simulated events, with at least one residual value exceeding the value of the corresponding threshold. Specifically, the events are detected at time instants $2.01 \mathrm{~s}, 2.01 \mathrm{~s}$ and $3.47 \mathrm{~s}$, for case studies $1 \mathrm{~A}, 1 \mathrm{~B}$, and $1 \mathrm{C}$, respectively, denoting in this way success of the anomaly detection strategy.
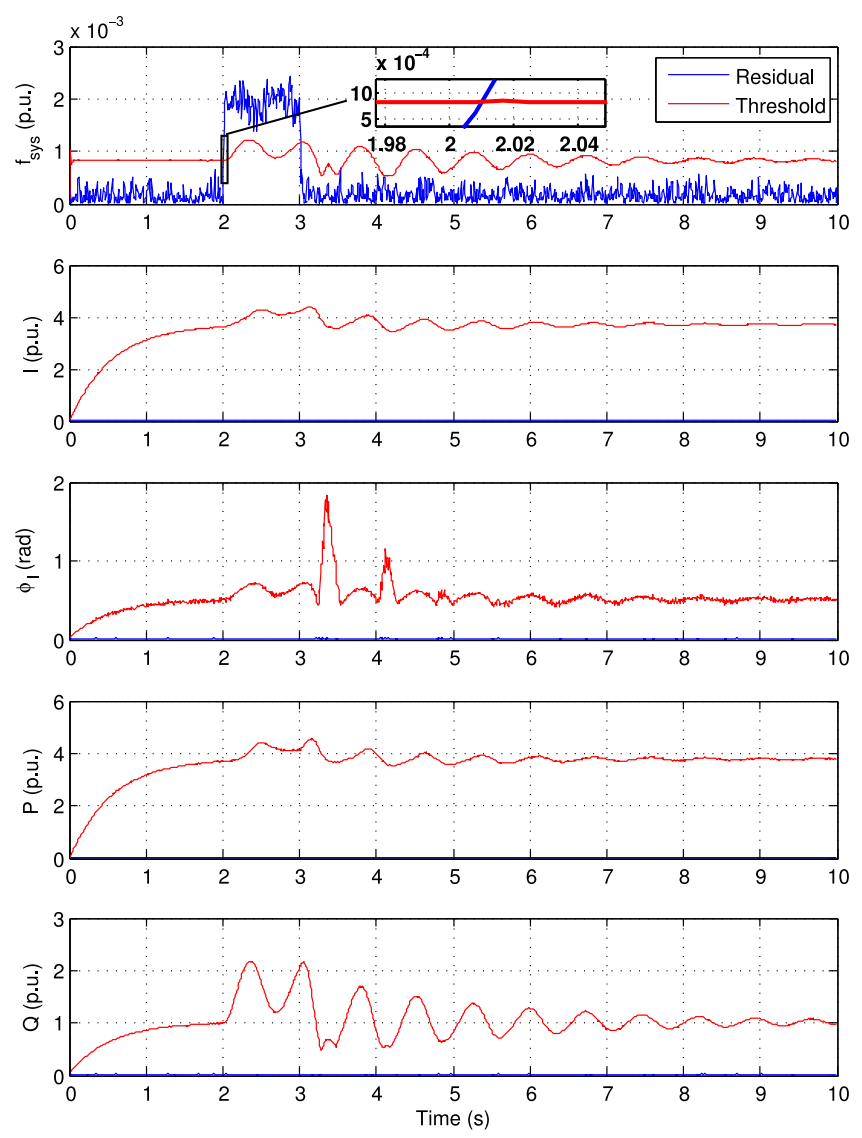

Fig. 4. Case Study 1B: Output residuals and thresholds for Gen. 2.

\section{B. IEEE Benchmark 68-Bus, 16-Machine System}

The successful performance of the suggested anomaly detection scheme on the previous study system, incentivized its application to a larger, realistic power system. For this purpose, the IEEE benchmark 68-bus, 16-machine NETS-NYPS system has been used [29], illustrated in Fig. 6. The details of this system can be found in [44]. Here, the synchronous machines, which are not characterized by manual excitation (i.e. the first 12), are equipped with fast static exciters, described by (7), to enable fast system response to contingencies, in a similar approach as in the previous system, and to facilitate the application of OELs [4]. The exciter design details are the same as in the previous study system. Moreover, same measurement noise levels as in the previous test system related case studies have been considered. Three case studies have been examined:

- Case Study 2A: A three-phase to ground fault occurs at bus 25 , at the time instant $t=2 \mathrm{~s}$, it is cleared after $100 \mathrm{~ms}$ and the line connecting buses 25 and 26 is tripped at the same time. The measurements are obtained at bus 25 , and the generator of interest is Gen. 8 .

- Case Study 2B: A step increase by 1 p.u. in $T_{m}$ of Gen. 5 occurs at the time instant $t=2 \mathrm{~s}$ and lasts for $1 \mathrm{~s}$, returning to its previous value afterwards. The measurements are obtained at bus 20 .

- Case Study 2C: The OEL of Gen. 6 gets activated at the time instant $t=3.34 \mathrm{~s}$. The OEL used is of takeover type, 

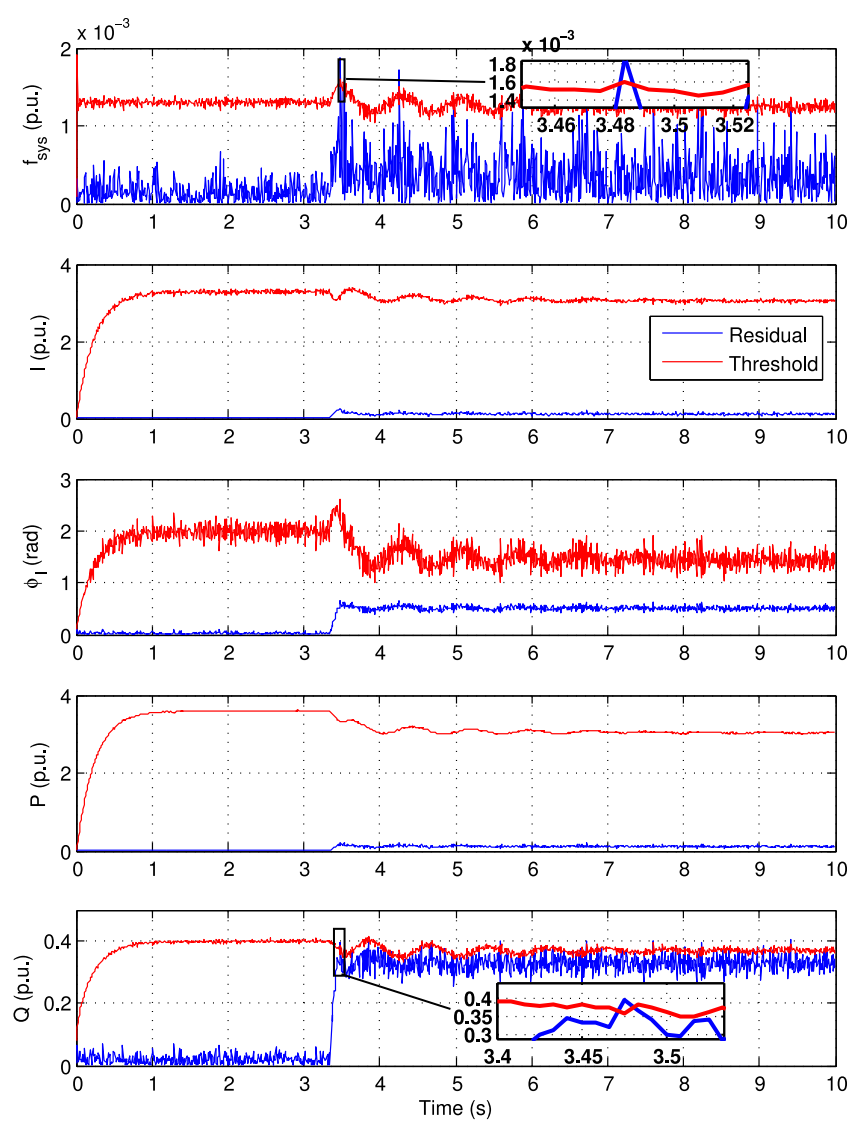

Fig. 5. Case Study 1C: Output residuals and thresholds for Gen. 1.

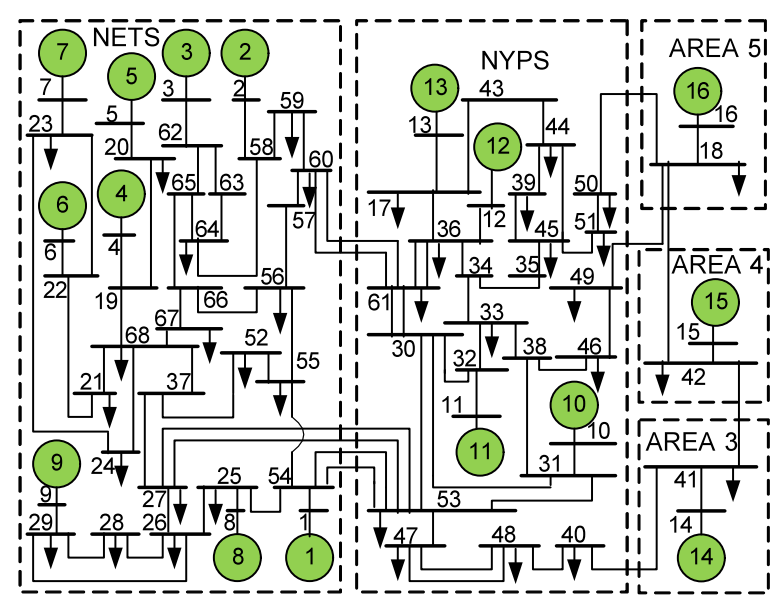

Fig. 6. NETS-NYPS 68-bus, 16-machine system.

designed according to the error signal substitution scheme, the design characteristics and the block diagram of which can be found in [4]. The measurements are obtained at bus 22 .

The implementation results depicting the residual and the threshold values for all measurement outputs of the case studies 2A, 2B, 2C, and Gens. 8, 5, 6, respectively, are shown in Figs. 7, 8 , and 9 , respectively. The success of the proposed anomaly detection algorithm can be easily evidenced, with the events being captured at time instants $2.01 \mathrm{~s}, 2.05 \mathrm{~s}$, and $3.37 \mathrm{~s}$, for case
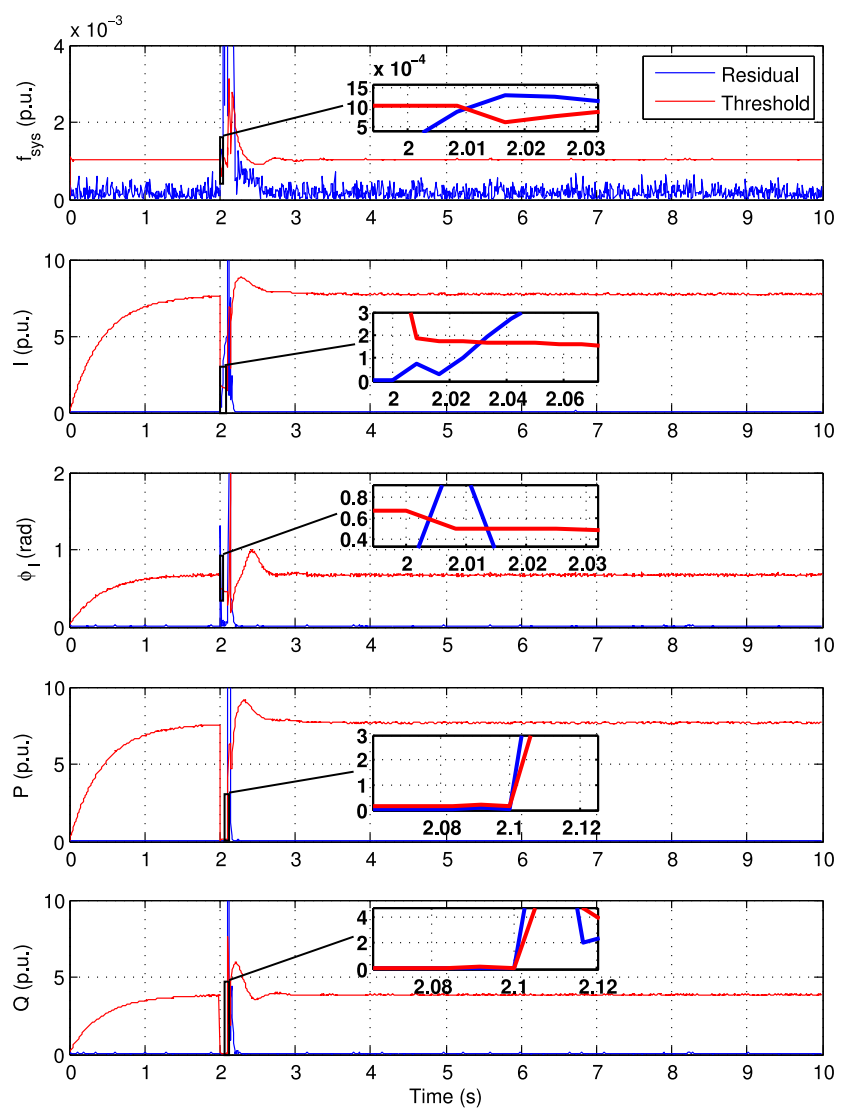

Fig. 7. Case Study 2A: Output residuals and thresholds for Gen. 8.
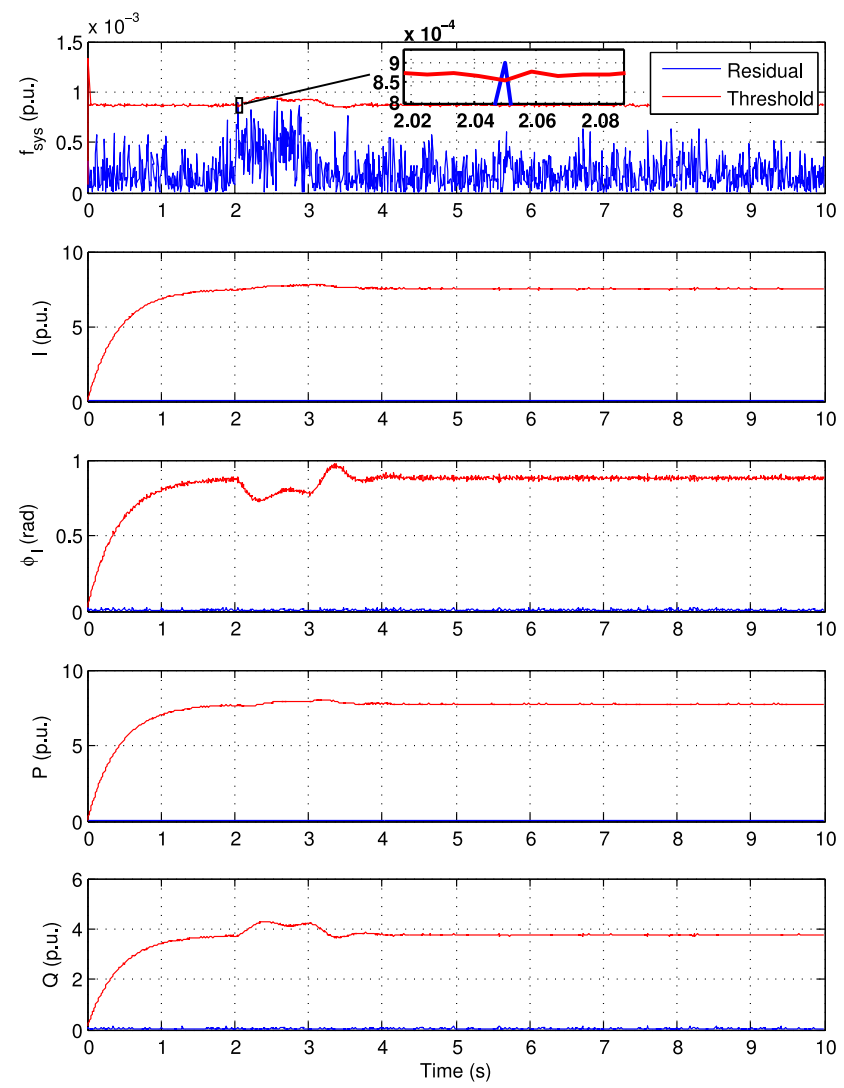

Fig. 8. Case Study 2B: Output residuals and thresholds for Gen. 5. 

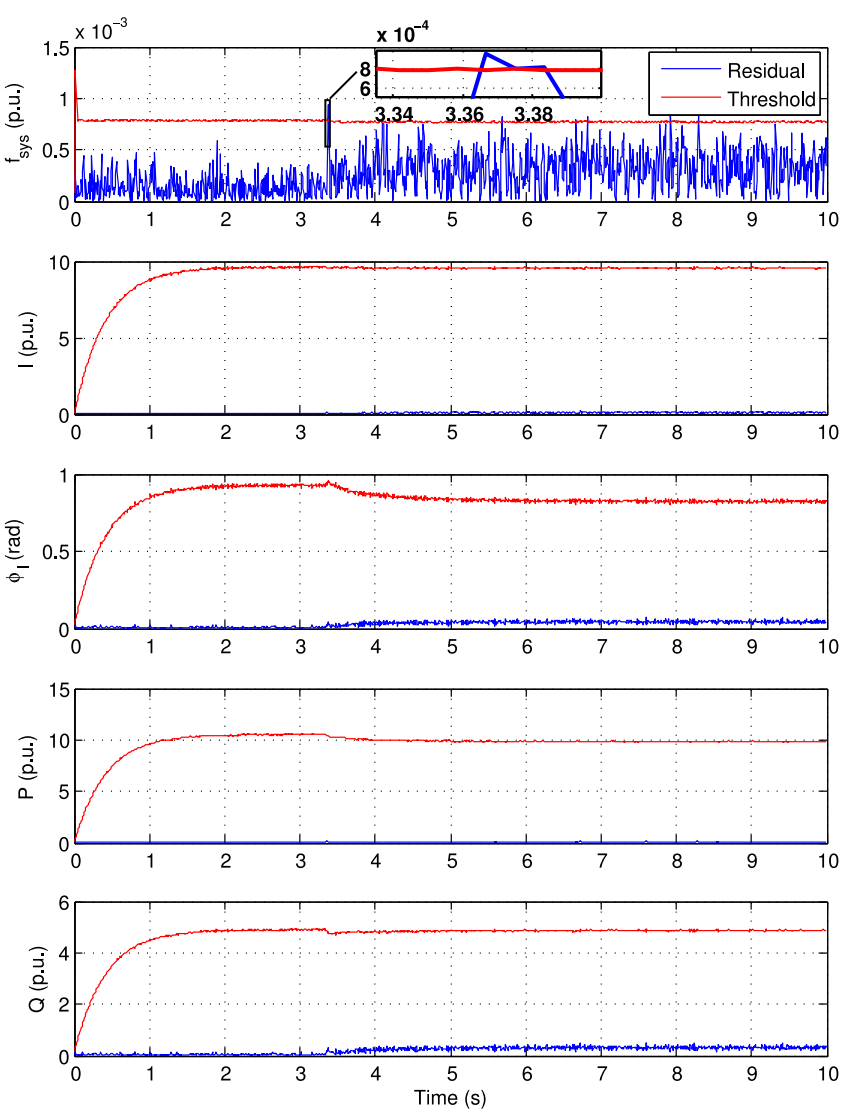

Fig. 9. Case Study 2C: Output residuals and thresholds for Gen. 6.

studies $2 \mathrm{~A}, 2 \mathrm{~B}$, and $2 \mathrm{C}$, respectively. It has to be noted that, the proposed anomaly detection strategy is able to capture the OEL operation, without the need for excitation voltage measurements, as in [4], where the practice of OEL activation detection is primarily based on excitation voltage measurements.

\section{CONCLUSION}

An observer-based anomaly detection scheme has been presented, which is able to trace deviations from nominal power system operation. This methodology relies on a linear timevarying observer, addressing the need for an estimation methodology tailored to a nonlinear system like a power network, and it can be implemented in real time. The method makes use of measurements at the high voltage side of the transformer connected to the terminal bus of the respective generator only, facilitating the utilization of a local approach. The proposed algorithm has been tested on two power system models, a small 9-bus 3-machine system, and the large, realistic IEEE benchmark 68-bus 16-machine system, where the results validate the success of its implementation. It is important to highlight that the anomaly detection scheme is based on a rigorous threshold calculation, without requiring the consideration of noise probability distributions or empirical criteria, making this method highly practical. This technique, being effective in detecting the activation of limiting devices such as overexcitation limiters, may serve as a valuable tool for model updates in the context of power system dynamic security assessment algorithms. The suggested methodology can prove to be extremely beneficial to operators in the context of power system monitoring, given the model uncertainty characterizing modern power network operation.

\section{APPENDIX A}

AnOMAly Detection Algorithm

The anomaly detection scheme is summarized in Alg. 1 .

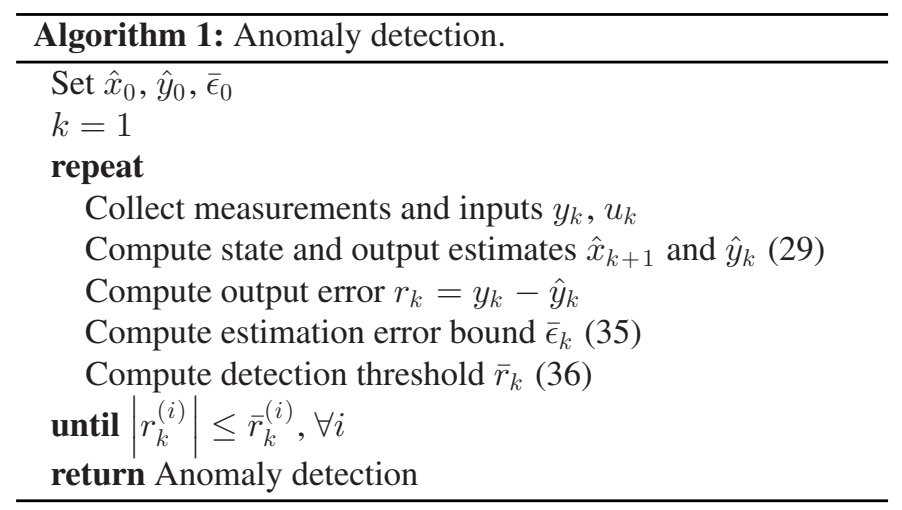

\section{APPENDIX B \\ CONVERGENCE ANALYSIS}

The proposed observer's (29) convergence analysis requires the consideration of the state estimation error, whose dynamics can be described by the following linear time-varying model:

$$
\begin{aligned}
\epsilon_{k+1} & =x_{k+1}-\hat{x}_{k+1} \\
& =A_{k} \epsilon_{k}-B_{k} \nu_{k}+w_{k}-K_{k}\left(y_{k}-\hat{y}_{k}\right) \\
& =\left(A_{k}-K_{k} C_{k}\right) \epsilon_{k}-B_{k} \nu_{k}+w_{k}-K_{k}\left(-D_{k} \nu_{k}+v_{k}\right) .
\end{aligned}
$$

In order to guarantee the convergence of the state estimation error (38), the result in [41] is used to define the matrix $K_{k}$ at each discrete time-step $k$ as:

$$
K_{k}=A_{k} \Phi_{k, k-t-1} G_{k, k-t-1}^{-1} \Phi_{k, k-t-1}^{\top} C_{k}^{\top},
$$

where $t$ is a positive constant integer, $\Phi_{k, k-t-1}$ is the transition matrix from time $k-t-1$ to time $k$ :

$$
\Phi_{k, k-t-1}=A_{k-1} A_{k-2} \ldots A_{k-t-1},
$$

with $\Phi_{k, k}=\mathbf{I}$, and $G_{k, k-t-1}$ is the observability Gramian, defined as:

$$
G_{k, k-t-1}=\sum_{l=k-t-1}^{k} \Phi_{l, k-t-1}^{\top} C_{l}^{\top} C_{l} \Phi_{l, k-t-1} .
$$

We have the following result:

Proposition B.1: The state estimation error (38), with the filtering matrix $K_{k}$ defined as in (39), represents the dynamics of a Bounded Input Bounded Output stable system.

Proof: In [41] it is demonstrated that the proposed filtering matrix $K_{k}$ allows to guarantee the exponentially asymptotically 
stability of the unforced system

$$
\epsilon_{k+1}=\left(A_{k}-K_{k} C_{k}\right) \epsilon_{k}
$$

under the assumption that the pair $\left[A_{k}, C_{k}\right]$ is uniform with respect to complete observability. Since the uncertainties are all bounded, then the time-varying system is Bounded Input Bounded Output stable.

Here, in all case studies, $t=1$, satisfying the uniform observability requirement [41].

\section{REFERENCES}

[1] K. Morison, L. Wang, and P. Kundur, "Power system security assessment," IEEE Power Energy Mag., vol. 2, no. 5, pp. 30-39, Sep. 2004.

[2] P. Panciatici, G. Bareux, and L. Wehenkel, "Operating in the fog: Security management under uncertainty," IEEE Power Energy Mag., vol. 10, no. 5 , pp. 40-49, Sep. 2012.

[3] P. Zhang, F. Li, and N. Bhatt, "Next-generation monitoring, analysis, and control for the future smart control center," IEEE Trans. Smart Grid, vol. 1, no. 2, pp. 186-192, Sep. 2010.

[4] G. Anagnostou and B. C. Pal, "Impact of overexcitation limiters on the power system stability margin under stressed conditions," IEEE Trans. Power Syst., vol. 31, no. 3, pp. 2327-2337, May 2016.

[5] N. Bretas, "An iterative dynamic state estimation and bad data processing," Int. J. Elect. Power Energy Syst., vol. 11, no. 1, pp. 70-74, 1989. [Online]. Available: http://www.sciencedirect.com/science/article/pii/ 0142061589900100

[6] J. K. Mandal, A. K. Sinha, and L. Roy, "Incorporating nonlinearities of measurement function in power system dynamic state estimation," IEE Proc. - Gener, Transmiss. Distrib., vol. 142, no. 3, pp. 289-296, May 1995.

[7] P. F. Odgaard, B. Lin, and S. B. Jorgensen, "Observer and data-drivenmodel-based fault detection in power plant coal mills," IEEE Trans. Energy Convers., vol. 23, no. 2, pp. 659-668, Jun. 2008.

[8] H. Li and L. Yu, "Abnormity handing for power system adaptive dynamic state estimation," in Proc. Int. Conf. Advanced Power Syst. Autom. Protection, Oct. 2011, vol. 2, pp. 929-934.

[9] F. Aminifar, M. Shahidehpour, M. Fotuhi-Firuzabad, and S. Kamalinia, "Power system dynamic state estimation with synchronized phasor measurements," IEEE Trans. Instrum. Meas., vol. 63, no. 2, pp. 352-363, Feb. 2014

[10] D. Simon, Optimal State Estimation: Kalman, H Infinity, and Nonlinear Approaches. Hoboken, NJ, USA: Wiley, 2006.

[11] V. Venkatasubramanian, R. Rengaswamy, S. N. Kavuri, and K. Yin, "A review of process fault detection and diagnosis: Part I: Quantitative modelbased methods," Comput. Chemical Eng., vol. 27, pp. 293-311, 2003.

[12] R. Patton, P. Frank, and D. Clark, Fault Diagnosis in Dynamic Systems: Theory and Application. Upper Saddle River, NJ, USA: Prentice-Hall, 1989.

[13] M. Blanke, M. Kinnaert, J. Lunze, and M. Staroswiecki, "Distributed fault diagnosis and fault-tolerant control," in Diagnosis and Fault-Tolerant Control. Berlin, Germany: Springer-Verlag, 2016, pp. 467-518.

[14] S. Riverso, F. Boem, G. Ferrari-Trecate, and T. Parisini, "Plug-and-play fault detection and control-reconfiguration for a class of nonlinear largescale constrained systems," IEEE Trans. Autom. Control, vol. 61, no. 12, pp. 3963-3978, Dec. 2016.

[15] I. Shames, A. M. Teixeira, H. Sandberg, and K. H. Johansson, "Distributed fault detection for interconnected second-order systems," Automatica, vol. 47, no. 12, pp. 2757-2764, 2011.

[16] V. Reppa, M. M. Polycarpou, and C. G. Panayiotou, "Decentralized isolation of multiple sensor faults in large-scale interconnected nonlinear systems," IEEE Trans. Autom. Control, vol. 60, no. 6, pp. 1582-1596, Jun. 2015.

[17] M. Davoodi, N. Meskin, and K. Khorasani, "Simultaneous fault detection and consensus control design for a network of multi-agent systems," Automatica, vol. 66, pp. 185-194, 2016.

[18] F. Boem, R. M. G. Ferrari, C. Keliris, T. Parisini, and M. M. Polycarpou, "A distributed networked approach for fault detection of large-scale systems," IEEE Trans. Autom. Control, vol. 62, no. 1, pp. 18-33, Jan. 2017.

[19] E. Tse, "Observer-estimators for discrete-time systems," IEEE Trans. Autom. Control, vol. 18, no. 1, pp. 10-16, Feb. 1973.
[20] Z. Hidayat, R. Babuska, B. D. Schutter, and A. Nez, "Observers for linear distributed-parameter systems: A survey," in Proc. IEEE Int. Symp. Robot. Sensors Environments, Sep. 2011, pp. 166-171.

[21] E. Scholtz, M. Larsson, and P. Korba, "Real-time parameter estimation of dynamic power systems using multiple observers," in Proc. IEEE Lausanne PowerTech., Jul. 2007, pp. 155-160.

[22] F. Caliskan and I. Genc, "A robust fault detection and isolation method in load frequency control loops," IEEE Trans. Power Syst., vol. 23, no. 4, pp. 1756-1767, Nov. 2008.

[23] N. Abu-Tabak, J. Y. Auloge, and P. Auriol, "Reduced-order observer design for small-signal multi-machine power system stability improvement by optimal control," in Proc. 2nd UKSIM Eur. Symp. Comput. Modeling Simul., Sep. 2008, pp. 370-375.

[24] E. Scholtz and B. C. Lesieutre, "Graphical observer design suitable for large-scale dae power systems," in Proc. 47th IEEE Conf. Decision Control, Dec. 2008, pp. 2955-2960.

[25] M. Esreraig and J. Mitra, "An observer-based protection system for microgrids," in Proc. IEEE Power Eng. Soc. Gen. Meet., Jul. 2011, pp. 1-7.

[26] S. Saha, M. Aldeen, and C. Tan, "Fault detection in transmission networks of power systems," Int. J. Elect. Power Energy Syst., vol. 33, no. 4, pp. 887900, 2011. [Online]. Available: http://www.sciencedirect.com/science/ article/pii/S0142061511000123

[27] F. Boem, R. M. G. Ferrari, T. Parisini, and M. M. Polycarpou, "Distributed fault diagnosis for continuous-time nonlinear systems: The input-output case," Annu. Rev. Control, vol. 37, no. 1, pp. 163-169, 2013.

[28] P. W. Sauer and M. A. Pai, Power System Dynamics and Stability. Englewood Cliffs, NJ, USA: Prentice-Hall, 1998. [Online]. Available: http://books.google.co.uk/books?id=dOOeAQAAIAAJ

[29] C. Canizares et al., "Benchmark models for the analysis and control of small-signal oscillatory dynamics in power systems," IEEE Trans. Power Syst., vol. 32, no. 1, pp. 715-722, Jan. 2017.

[30] J. Machowski, J. Bialek, and J. Bumby, Power System Dynamics and Stability. Hoboken, NJ, USA: Wiley, 1997. [Online]. Available: http://books.google.co.uk/books?id=L7o4TitajRIC

[31] Z. Huang, P. Du, D. Kosterev, and S. Yang, "Generator dynamic model validation and parameter calibration using phasor measurements at the point of connection," IEEE Trans. Power Syst., vol. 28, no. 2, pp. 19391949, May 2013.

[32] A. Singh and B. Pal, "Decentralized dynamic state estimation in power systems using unscented transformation," IEEE Trans. Power Syst., vol. 29, no. 2, pp. 794-804, Mar. 2014.

[33] G. Anagnostou and B. C. Pal, "Derivative-free Kalman filtering based approaches to dynamic state estimation for power systems with unknown inputs," IEEE Trans. Power Syst., vol. 33, no. 1, pp. 116-130, Jan. 2018.

[34] E. Ghahremani and I. Kamwa, "Local and wide-area pmu-based decentralized dynamic state estimation in multi-machine power systems," IEEE Trans. Power Syst., vol. 31, no. 1, pp. 547-562, Jan. 2016.

[35] E. Ghahremani and I. Kamwa-, "Dynamic state estimation in power system by applying the extended Kalman filter with unknown inputs to phasor measurements," IEEE Trans. Power Syst., vol. 26, no. 4, pp. 2556-2566, Nov. 2011

[36] A. A. Hajnoroozi, F. Aminifar, and H. Ayoubzadeh, "Generating unit model validation and calibration through synchrophasor measurements," IEEE Trans. Smart Grid, vol. 6, no. 1, pp. 441-449, Jan. 2015.

[37] L. Fan and Y. Wehbe, "Extended Kalman filtering based real-time dynamic state and parameter estimation using PMU data," Electr. Power Syst. Res., vol. 103, pp. 168-177, 2013. [Online]. Available: http://www.sciencedirect.com/science/article/pii/S0378779613001442

[38] B. Pal and B. Chaudhuri, Robust Control in Power Systems. Berlin, Germany: Springer-Verlag, 2006.

[39] M. Pavella, D. Ernst, and D. Ruiz-Vega, Transient Stability of Power Systems: A Unified Approach to Assessment and Control (Kluwer International Series in Engineering and Computer Science). Berlin, Germany: Springer-Verlag, 2000. [Online]. Available: http://books.google.co.uk/ books?id=qhl0OusNAnsC

[40] A. G. Phadke and B. Kasztenny, "Synchronized phasor and frequency measurement under transient conditions," IEEE Trans. Power Del., vol. 24, no. 1, pp. 89-95, Jan. 2009.

[41] J. Moore and B. Anderson, "Coping with singular transition matrices in estimation and control stability theory," Int. J. Control, vol. 31, no. 3, pp. 571-586, 1980.

[42] IEEE Standard for Synchrophasor Measurements for Power Systems, IEEE Standard C37.118.1-2011 (Revision of IEEE Standard C37.1182005), Dec. 2011, pp. 1-61. 
[43] B. Anderson and J. B. Moore, "Detectability and stabilizability of timevarying discrete-time linear systems," SIAM J. Control Optimization, vol. 19, no. 1, pp. 20-32, 1981.

[44] NETS-NYPS 68 Bus System. [Online]. Available: http://www.sel.eesc. usp.br/ieee/

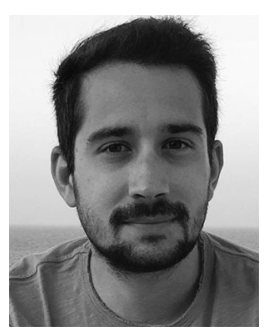

Georgios Anagnostou (M'17) was born in Athens, Greece. He received the Diploma in electrical and computer engineering from the National Technical University of Athens, Athens, Greece, the M.Sc. degree in sustainable energy futures from Imperial College London, London, U.K., and the Ph.D. degree in electrical engineering from Imperial College London, in 2011, 2012, and 2016, respectively. His current research interests include power system dynamics and control, dynamic security assessment, dynamic state estimation, and renewable energy based generation.

Dr. Anagnostou is a member of the Technical Chamber of Greece.

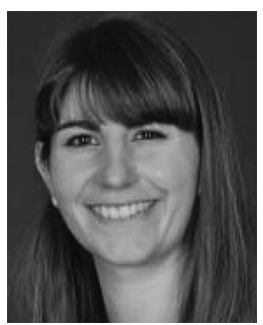

Francesca Boem received the M.Sc. degree (cum laude) in management engineering and the Ph.D. degree in information engineering from the University of Trieste, Trieste, Italy, in 2009 and 2013, respectively. From 2013 to 2014, she was a Postdoctoral Fellow in the Department of Engineering and $\mathrm{Ar}$ chitecture, University of Trieste with the Machine Learning Group. Since 2014, she has been working as a Research Associate in the Department of Electrical and Electronic Engineering, Imperial College London, London, U.K. with the Control and Power Research Group. In 2015-2016, she was part of the team at Imperial College London working on the Phase I of the flagship EU H2020-WIDESPREADTEAMING project for the development of the KIOS Research and Innovation Centre of Excellence, a strategic partnership between University of Cyprus and Imperial College London, which has been successful for Phase II. Her current research interests include distributed fault diagnosis and fault-tolerant control methods for large-scale networked systems and distributed estimation methods for sensor networks.

Dr. Boem is a Member of the IFAC Technical Committee 6.4 (Fault Detection, Supervision \& Safety of Technical Processes-SAFEPROCESS) and an Associate Editor for the IEEE Control System Society Conference Editorial Board and for the EUCA Conference Editorial Board.

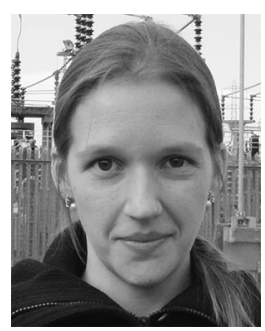

Stefanie Kuenzel (GS'11-M'14) received the M.Eng. and Ph.D. degrees from Imperial College London, London, U.K., in 2010 and 2014, respectively. She is currently the Head of the Power Systems group and a Lecturer in the Department of Electronic Engineering, Royal Holloway, University of London, London, U.K., and a Visiting Researcher at Imperial College London. Her current research interests include renewable generation and transmission, including HVDC.

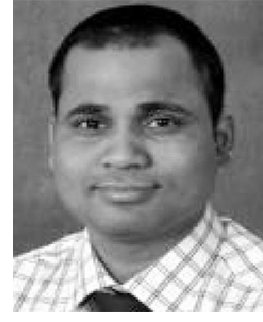

Bikash C. Pal (M'00-SM'02-F'13) received the B.E.E. with honors degree in electrical engineering from Jadavpur University, Calcutta, India, the M.E. degree in electrical engineering from the Indian Institute of Science, Bangalore, India, and the Ph.D. degree in electrical engineering from Imperial College London, London, U.K, in 1990, 1992, and 1999, respectively. He is currently a Professor in the Department of Electrical and Electronic Engineering, Imperial College London. His current research interests include state estimation, power system dynamics, and flexible AC transmission system controllers.

Prof. Pal is the Editor-in-Chief of IEEE TRANSACtions on Sustainable ENERGY and a Fellow of the IEEE for his contribution to power system stability and control.

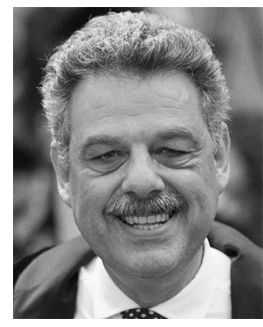

Thomas Parisini (F'11) received the Ph.D. degree in electronic engineering and computer science from the University of Genoa, Italy, in 1993. He holds the Chair of Industrial Control and is the Director of Research at Imperial College London, London, U.K. Since 2001, he has been the Danieli Endowed Chair of Automation Engineering with the University of Trieste, Trieste, Italy. He authored or co-authored more than 270 research papers in archival journals, book chapters, and international conference proceedings. His research interests include neural-network approximations for optimal control problems, fault diagnosis for nonlinear and distributed systems, nonlinear model predictive control systems, and nonlinear estimation.

Prof. Parisini is a co-recipient of the 2011-2013 IFAC Best Application Paper Prize of the Journal of Process Control, and of the 2004 Outstanding Paper Award of the IEEE TRANSACTIONS ON NEURAL NetworKs. He received the 2007 IEEE Distinguished Member Award. In 2016, he was awarded as PI at Imperial the H2020 Flagship Teaming Project KIOS (total budget for the two partners over 40 MEuros). In 2012, he received the prestigious ABB Research Grant dealing with energy-autonomous sensor networks for self-monitoring industrial environments. He was the Editor-in-Chief of the IEEE TRANSACTIONS ON CONTROL SYSTEMS TECHNOLOGY and is currently serving as the Vice President for Publications Activities of the IEEE Control Systems Society. He is also the Chair of the IFAC Technical Committee on Fault Detection, Supervision \& Safety of Technical Processes. He was the Chair of the IEEE CSS Conference Editorial Board and a Distinguished Lecturer of the IEEE. He is currently serving as an Editor for Control Application of Automatica. He is also an Associate Editor of the International Journal of Control and an Associate Editor of the IEEE TRANSACTIONS ON AUTOMATIC CONTROL, the IEEE TRANSACTIONS ON NeURAL NeTwORKS, Automatica, and the International Journal of Robust and Nonlinear Control. He was the Program Chair of the 2008 IEEE CDC and a General Co-Chair of the 2013 IEEE CDC. He is a Fellow of the IFAC. 